A-riviog

Free to Authors and Readers

\section{A Platinum Open Access Journal}

for Organic Chemistry

DOAJ Seal
Paper

Arkivoc 2021, part iii, 67-75

\title{
Halogen-bonding in 3-nitrobenzaldehyde-derived dichlorodiazadienes
}

Namiq G. Shikhaliyev, ${ }^{a *}$ Abel M. Maharramov, ${ }^{a}$ Gulnar T. Suleymanova, ${ }^{a}$ Gulnara V. Babayeva, ${ }^{a}$ Gunay Z. Mammadova, ${ }^{a}$ Irada M. Shikhaliyeva, ${ }^{a}$ Aliyar A. Babazade, ${ }^{a}$ and Valentine G. Nenajdenko*b

a Organic Chemistry Department, Baku State University, Z. Xalilov Str. 23, Az 1148 Baku, Azerbaijan

${ }^{b}$ Department of Chemistry, M. V. Lomonosov Moscow State University, 1 Leninskie Gory, 119992 Moscow, Russian Federation

Email:namiqst@gmail.com,nenajdenko@gmail.com

In honor of Professor Lanny Liebeskind

Received 10-26-2020
Published on line 01-05-2021

\section{Abstract}

A set of 4,4-dichloro-1,2-diazabutadienes derived from 3-nitrobenzaldehyde was prepared by the copper catalyzed reaction of the corresponding hydrazones with $\mathrm{CCl}_{4}$ in the presence of TMEDA. The structure of all products was confirmed by ${ }^{1} \mathrm{H}$ and ${ }^{13} \mathrm{C}$ NMR spectra and ESI-MS. X-ray diffraction revealed $\mathrm{Cl} \cdots \mathrm{O}$ halogen binding in the crystal form for some dienes. Moreover, $\mathrm{Br} \cdots \pi$ and $\mathrm{Cl} \cdot \pi$ types of halogen bonds are observed. Azo dyes absorb in the UV-Vis region with the $\lambda_{\text {max }}$ located at about $234-437 \mathrm{~nm}$ in $\mathrm{CH}_{2} \mathrm{Cl}_{2}, \mathrm{DMF}$ and $\mathrm{MeOH}$. Both absorption intensity and $\lambda_{\max }$ is dependent on the solvent polarity and the electronwithdrawing/donating ability of para-substituents on the aromatic moiety of the dichlorodiazadienes.

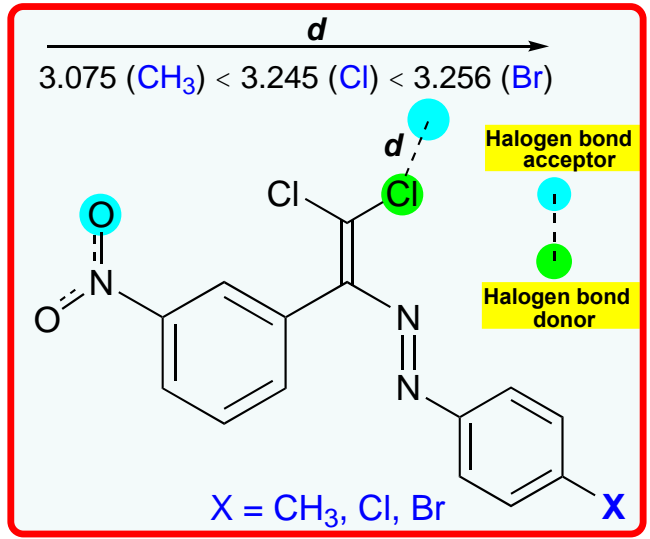

Keywords: Weak interactions, halogen bonding; hydrogen bonding; azo dyes, olefination 


\section{Introduction}

Azo dyes $\mathrm{R}-\mathrm{N}=\mathrm{N}-\mathrm{R}(\mathrm{R}=$ aryl, heterocycle, etc.) are a well-known class of organic compounds in synthetic chemistry. They have been extensively used for ink jet printing, as food additives, for dyeing textiles, as indicators, cosmetics, in non-linear optics and dye-sensitized solar cells, as pigments, radical reaction initiators, photo-sensitizers, and therapeutic agents, etc. ${ }^{1-5}$ Due to the industrial applications of azo dyes, the $-\mathrm{N}=\mathrm{N}$ - fragment has been incorporated into polymers and supramolecular compounds to show valuable properties and the possibility of fine-tuning by variation of R. 6,7 There are many synthetic strategies for the synthesis of azo compounds, but the well-established method is azo-coupling of an aryl diazonium cation with another aryl ring, especially those substituted with electron-donating groups. ${ }^{1,2}$ Recently, some of us have developed a new method for the synthesis of azo dyes, namely a copper-catalyzed transformation of $\mathrm{N}$ substituted hydrazones into halogenated azabutadienes. ${ }^{8}$ The attached reactive olefin moiety can contribute to $\pi$-conjugation in the design of functional materials or used for further functionalization of dyes.

Due to high directionality, halogen bonding has been used in the design of materials, catalysis and crystal engineering similarly to chalcogen ${ }^{9}$ and pnictogen bonds. ${ }^{10-15}$ Halogen bonding is also an effective strategy to control molecular recognition in solution. ${ }^{16}$ The study of halogen-bonded associates has become one of the most attractive areas of research in the solution chemistry. ${ }^{17}$ However, to the best of our knowledge, the role of halogen bonding in azo chemistry remains unexplored in spite of high activity in this field. ${ }^{18-22}$ Herein, we demonstrate that this type of noncovalent interaction is of great importance in the design of diazadienes in terms of their intermolecular halogen bond strength (Scheme 1). The para-substituents on the aromatic moiety range from typical electron-withdrawing $(-\mathrm{Br},-\mathrm{Cl}$ and $-\mathrm{F})$ to typical electron-donating groups $\left(-\mathrm{OCH}_{3}\right.$ and $\left.-\mathrm{CH}_{3}\right)$.

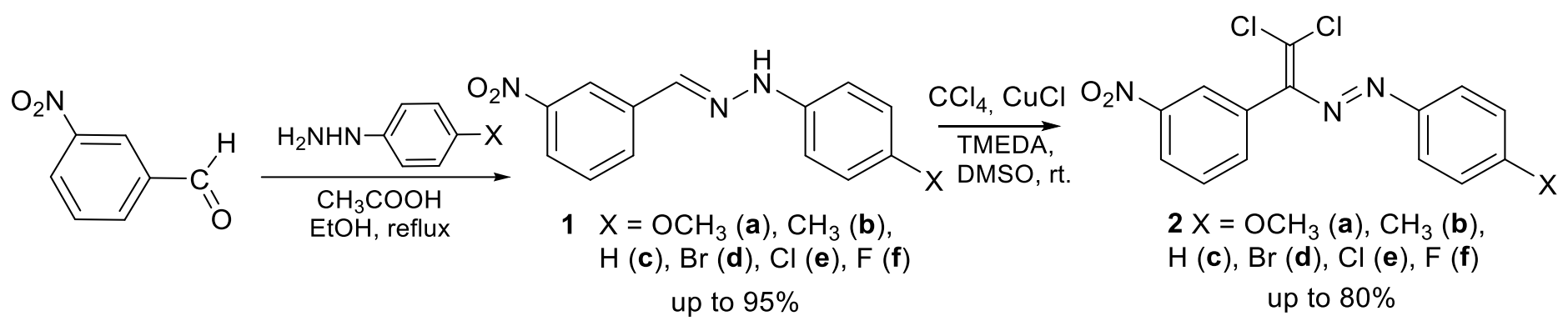

Scheme 1. Synthesis of diazadienes 2.

\section{Results and Discussion}

A set of hydrazones 1 was prepared by the reaction of 3-nitrobenzaldehyde with hydrochlorides of 4substituted aryl hydrazines (Scheme 1). The reaction was performed in the presence of $\mathrm{CH}_{3} \mathrm{COONa}$ in $\mathrm{EtOH}$ at $80{ }^{\circ} \mathrm{C}$ [see Electronic Supplementary Information (ESI)]. Next, the reaction of 1 with $\mathrm{CCl}_{4}$ in the presence of $\mathrm{CuCl}$ and tetramethylethylenediamine (TMEDA) in DMSO permitted synthesis of the corresponding dichlorodiazadienes $\mathbf{2}$. The prepared dienes 2 were isolated as $E$-isomers in up to $80 \%$ yield (see ESI). The structures of all products were confirmed by ${ }^{1} \mathrm{H}$ and ${ }^{13} \mathrm{C}$ NMR spectra and ESI-MS. All diazadienes 2 have characteristic signals in their ${ }^{13} \mathrm{C}$ NMR spectra at $123-125 \mathrm{ppm}$ which are typical for these compounds. ${ }^{8}$ The peaks corresponding to the aryl rings of dienes $\mathbf{2}$ were found in the NMR spectra. In addition, X-ray data were 
obtained for some products (Figure 1). Single crystal X-ray analyses of $\mathbf{2 b}, \mathbf{2 d}$ and $\mathbf{2 e}$ show that the 3-nitroaromatic moiety rotates around the $\mathrm{C} 1-\mathrm{C} 7$ bond with dihedral angles of $65.0(5)^{\circ} \mathrm{C} 6-\mathrm{C} 1-\mathrm{C} 7-\mathrm{N} 1,114.0(2)^{\circ}$ $\mathrm{C} 2-\mathrm{C} 1-\mathrm{C} 7-\mathrm{N} 1$ and $115.4(2)^{\circ} \mathrm{C} 2-\mathrm{C} 1-\mathrm{C} 7-\mathrm{N} 1$ respectively, whereas azo $\mathrm{N}=\mathrm{N}$ and olefin $\mathrm{C}=\mathrm{C}$ fragments are quasi planar with small deviations (Figure 1). Compounds $\mathbf{2} \mathbf{b}, \mathbf{2} \mathbf{d}$ and $\mathbf{2 e}$ contain the $\mathbf{C}-\mathrm{Cl} \cdots \mathrm{O}$ type of intermolecular halogen bonds with distances of 3.075(4), 3.256(3) and 3.245(2) $\AA$, respectively, which are shorter than the sum of the Bondi's Van der Waals radii of the interacting atoms $\left(\mathrm{Cl}+\mathrm{O}=1.75+1.52=3.30 \AA^{23}\right)$ (Figure 2$)$. An attachment of halogen bond donor centers $\mathrm{Br}$ or $\mathrm{Cl}$ to the para-position of the aromatic moiety of $\mathbf{2 d}$ and $\mathbf{2 e}$ lead to $\mathrm{Br} \cdots \pi$ or $\mathrm{Cl} \cdots \pi$ types of intermolecular interactions with distances of 3.900 and $3.873 \AA$, respectively (Figure 2). Besides, the intermolecular $\mathrm{Cl} \cdots \mathrm{O}, \mathrm{Br} \cdots \pi$ and $\mathrm{Cl} \cdots \pi$ types of halogen bonds, all structures are stabilized by multiple weak intermolecular hydrogen bonds.

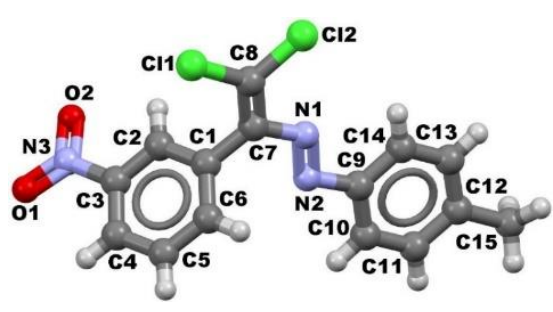

$2 b$

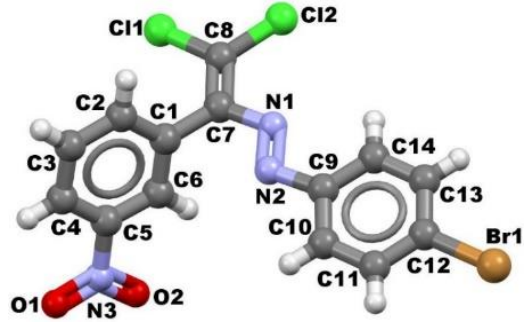

2d

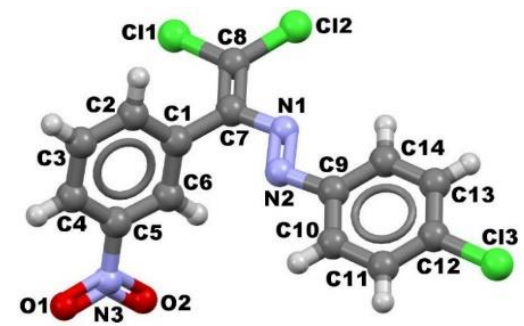

$2 e$

Figure 1. Molecular structures of $\mathbf{2 b}, \mathbf{2} \mathbf{d}$ and $\mathbf{2 e}$ (50\% ellipsoids) along with the atomic numbering schemes.
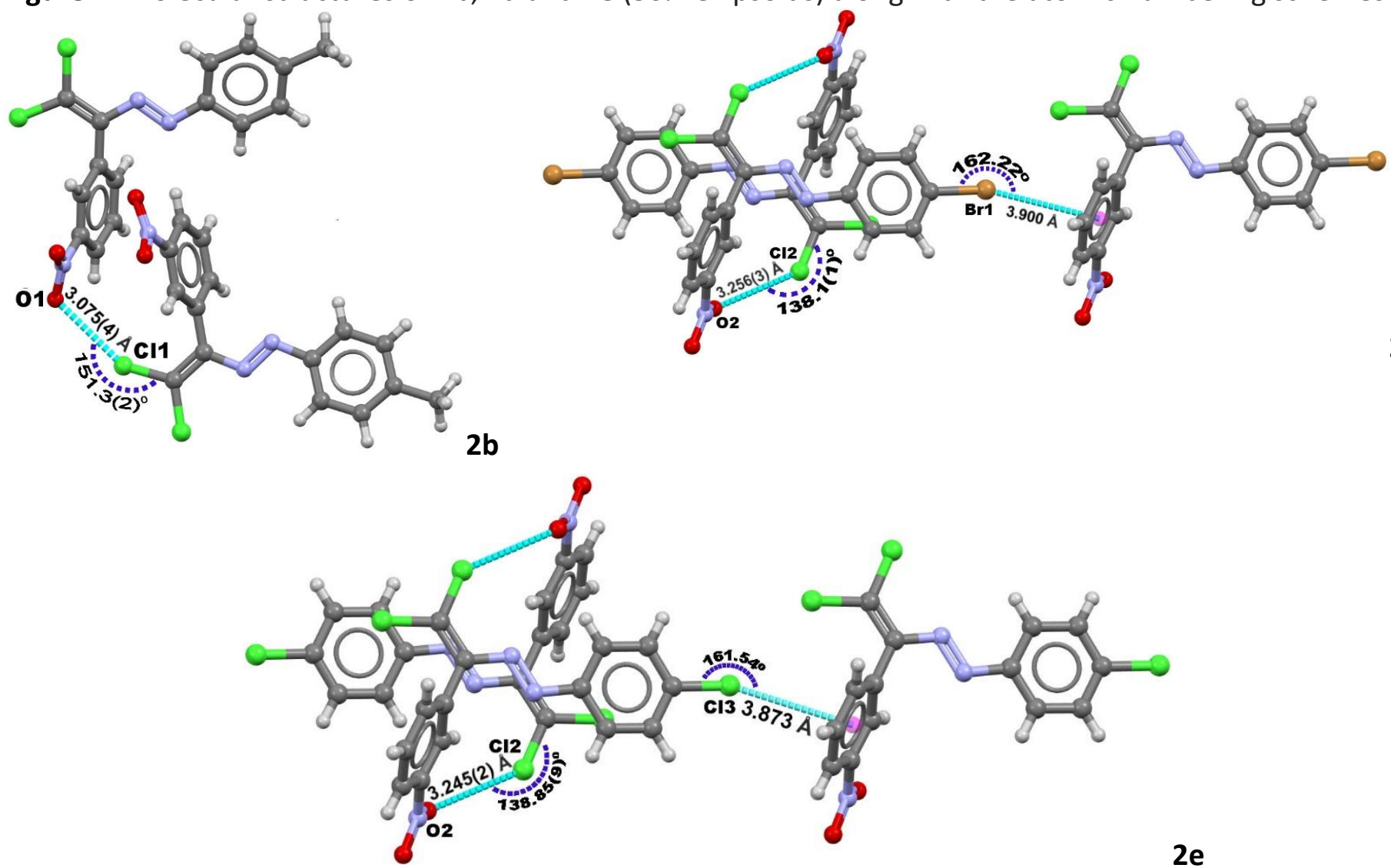

$2 e$

Figure 2. Intermolecular halogen bonds in $\mathbf{2 b}, \mathbf{2} \mathbf{d}$ and $\mathbf{2 e}$ (shown as dashed light blue line). 
Table. Absorption maxima ( $\lambda_{\max }$ ) of 2 in $\mathrm{CH}_{2} \mathrm{Cl}_{2}(\mathrm{a})$, DMF (b) and $\mathrm{MeOH}(\mathrm{c})$

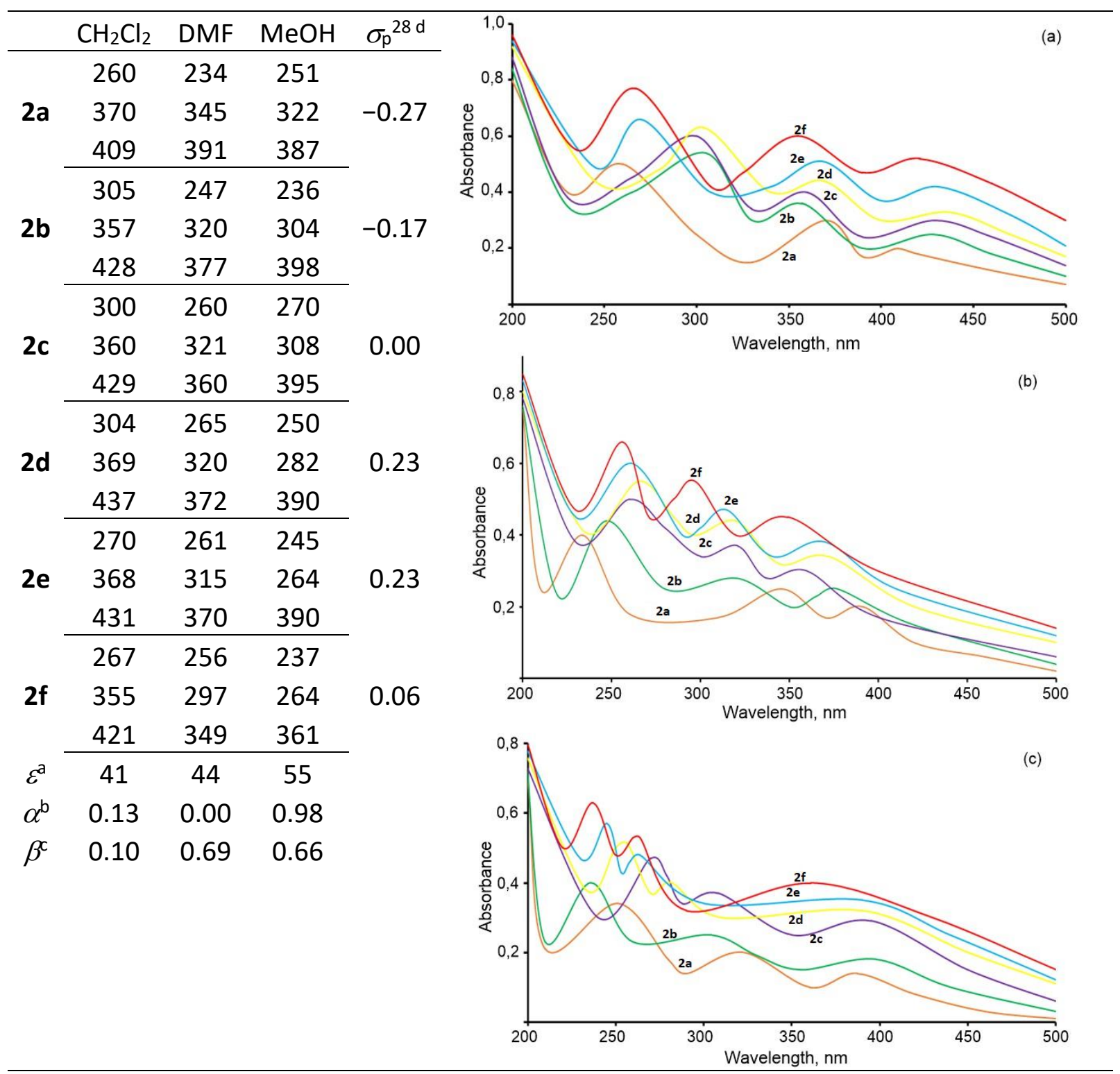

a Electric permittivity; ${ }^{\mathrm{b}} \mathrm{H}$-bond donating ability of solvent; ${ }^{24} \mathrm{c} \mathrm{H}$-bonding acceptor ability of solvent. ${ }^{24 \mathrm{~d}}$ Hammet substituent constant.

UV-visible absorption spectra of dienes 2 were recorded over the wavelength range of 200-500 $\mathrm{nm}$, in different solvents [dichloromethane $\left(\mathrm{CH}_{2} \mathrm{Cl}_{2}\right)$, dimethylformamide (DMF) and methanol (MeOH)] with different polarity indices, at $298 \mathrm{~K}$ and a concentration of $1.00 \cdot 10^{-6} \mathrm{~mol} \mathrm{~L}^{-1}$ (Table, Figure 3 ). Three absorption peaks were observed for all compounds (Figure 3), which are significantly influenced by the polarity, $\mathrm{H}$-bond donor or $\mathrm{H}$-bond acceptor ability $(\alpha \text { or } \beta)^{24}$ of the organic solvents and the electron-withdrawing or electrondonating character of the substituents. ${ }^{23}$ The shortest wavelength peak observed in the range of 234-305 $\mathrm{nm}$ can be assigned to the excitation of the $\pi$-electrons within the $\mathrm{C}=\mathrm{C}$ bonds in the olefin moieties. ${ }^{26-29}$ The second UV absorption peaks around 355-370 (in $\mathrm{CH}_{2} \mathrm{Cl}_{2}$ ), 297-345 (in DMF) and 264-322 (in $\mathrm{MeOH}$ ) $\mathrm{nm}$ (Table) can be attributed to the $\pi \rightarrow \pi^{*}$ transition in the aromatic rings. ${ }^{26-29}$ The third maxima in the UV spectra of 2a-2f in the range 409-437 (in $\mathrm{CH}_{2} \mathrm{Cl}_{2}$ ), 349-391 (in DMF) and 361-398 (in $\mathrm{MeOH}$ ) $\mathrm{nm}$ can be attributed to the $n \rightarrow \pi^{*}$ transition in $\mathrm{N}=\mathrm{N}$ bonds as well as intermolecular hydrogen, $\mathrm{Cl} \cdots \mathrm{O}, \mathrm{Br} \cdots \pi$ or $\mathrm{Cl} \cdots \pi$ types of halogen bonds. The absorption intensity of the studied dyes increases with a decrease of the solvent polarity, $\mathrm{MeOH}<$ 
DMF $<\mathrm{CH}_{2} \mathrm{Cl}_{2}$ (Figure 3), featuring a short energy gap between two states (ground and excited state) with strong intermolecular halogen bonding in $\mathrm{CH}_{2} \mathrm{Cl}_{2}$, whereas there is no clear trend/correlation between $\lambda_{\text {max }}$ and $\sigma_{\mathrm{p}}$ of para-substituents.

\section{Conclusions}

A set of (E)-1-(2,2-dichloro-1-(3-nitrophenyl)vinyl)-2-(para-substituted phenyl)diazenes 2 was synthesized by $\mathrm{Cu}$-catalysed olefination of the corresponding $\mathrm{N}$-aryl hydrazones with $\mathrm{CCl}_{4}$ in the presence of TMEDA in DMSO. Depending on the attached para-substituents in the $\mathrm{N}$-aryl fragment, $\mathrm{Cl} \cdots \mathrm{O}, \mathrm{Br} \cdots \pi$ and $\mathrm{Cl} \cdot \pi$ types of halogen bonds were found in these dyes. The UV spectra of 2 showed three $\lambda_{\max }$ in $\mathrm{CH}_{2} \mathrm{Cl}_{2}$, DMF and $\mathrm{MeOH}$, which can be attributed: $i$ ) the excitation of the $\pi$-electrons within the $\mathrm{C}=\mathrm{C}$ bonds; ii) the $\pi \rightarrow \pi^{*}$ transition in the aromatic rings; iii) the $n \rightarrow \pi^{*}$ transition in $\mathrm{N}=\mathrm{N}$ bonds as well as intermolecular halogen and hydrogen bonds. The electron-donating or -withdrawing character of para-substituents on the aromatic moiety was found to have significant impacts on optical properties of $\mathbf{2}$. According to the obtained results we can conclude that the attached halogen bond donor centers on dye molecules $\mathbf{2}$ may be useful for molecular recognition and design of functional materials. ${ }^{30-35}$

\section{Experimental Section}

General. All the chemicals were obtained from commercial sources (Aldrich) and used as received. Infrared spectra (4000-400 cm $\mathrm{cm}^{-1}$ ) were recorded on a Vertex 70 (Bruker) instrument in $\mathrm{KBr}$ pellets. Carbon, hydrogen, and nitrogen elemental analyses were carried out using a $2400 \mathrm{CHN}$ Elemental Analyzer (Perkin Elmer). The ${ }^{1} \mathrm{H}$ and ${ }^{13} \mathrm{C}$ NMR spectra were recorded on Bruker Advance II+ 300.13 (75.468 carbon-13) MHz (UltraShield ${ }^{\mathrm{TM}}$ Magnet) spectrometer at ambient temperature. Chemical shifts are reported in ppm using tetramethylsilane as the internal reference. Electrospray mass spectra (ESI-MS) were run with an ion-trap instrument (Varian 500-MS LC Ion Trap Mass Spectrometer) equipped with an electrospray ion source. For electrospray ionization, the drying gas and flow rate were optimized according to the particular sample with 35 p.s.i. nebulizer pressure. Scanning was performed from $\mathrm{m} / \mathrm{z} 0$ to 1100 in methanol solution. The compounds were observed in the positive mode (capillary voltage $=80-105 \mathrm{~V}$ ). The UV-vis absorption spectra in the $200-500$ $\mathrm{nm}$ regions were recorded with a scan rate of $240 \mathrm{~nm} \cdot \mathrm{min}^{-1}$ by using a Lambda 35 UV-vis spectrophotometer (Perkin-Elmer) in $1.00 \mathrm{~cm}$ quartz cells at room temperature, with a concentration of $2 \mathrm{a}-2 \mathrm{f}$ of $1.00 \cdot 10^{-6} \mathrm{~mol} \mathrm{~L}^{-1}$ in $\mathrm{CH}_{2} \mathrm{Cl}_{2}$, DMF or $\mathrm{MeOH}$.

$\mathbf{X}$-ray structure determinations. X-ray diffraction patterns of $\mathbf{2} \mathbf{b}, \mathbf{2} \mathbf{d}$ and $\mathbf{2} \mathbf{e}$ were collected using a Bruker SMART APEX-II CCD area detector equipped with graphite-monochromated Mo-Ka radiation $(\lambda=0.71073 \AA)$ at 296(2) K, respectively. Absorption correction was applied by SADABS [s1,s2]. The structures were solved by direct methods and refined on $F^{2}$ by full-matrix least-squares using Bruker's SHELXTL-97 [s3]. All non-hydrogen atoms were refined anisotropically. The hydrogen atoms were inserted at calculated positions. The details of the crystallographic data for $\mathbf{2} \mathbf{b}, \mathbf{2} \mathbf{d}$ and $\mathbf{2 e}$ are summarized in Table 1 . Crystallographic data for the structural analysis have been deposited to the Cambridge Crystallographic Data Center (CCDC 1897671 for 2b, 2019413 for $\mathbf{2 d}$ and 2019414 for $\mathbf{2 e}$ ). Copy of this information can be obtained free of charge from The Director, CCDC, 
12 Union Road, Cambridge CB2 1EZ, UK (Fax: (+44) 1223-336033; E-mail: deposit@ccdc.cam.ac.uk or www.ccdc.cam.ac.uk/data request/cif).

\section{Synthesis of 1a-1f}

A mixture of 3-nitrobenzaldehyde $(10 \mathrm{mmol}), \mathrm{CH}_{3} \mathrm{COONa}(10 \mathrm{mmol}), \mathrm{EtOH}(50 \mathrm{~mL})$ and the corresponding (para-substituted aryl hydrazine hydrochloride $(10.2 \mathrm{mmol})$ was heated at reflux at $80{ }^{\circ} \mathrm{C}$ with stirring for $2 \mathrm{~h}$. The reaction mixture was cooled to $\mathrm{rt}$ and $\mathrm{H}_{2} \mathrm{O}(50 \mathrm{~mL})$ was added to give a precipitate of crude product, which filtered off, washed with diluted $\mathrm{EtOH}$ (1:1 with water) and dried in vacuo on a rotary evaporator.

(E)-1-(4-Methoxyphenyl)-2-(3-nitrobenzylidene)hydrazine (1a). Red solid (90\%); mp 125-129 ${ }^{\circ} \mathrm{C}$, lit 130.5 ${ }^{\circ} \mathrm{C} .{ }^{36}$ Anal. Calcd for $\mathrm{C}_{14} \mathrm{H}_{13} \mathrm{~N}_{3} \mathrm{O}_{3}(M=271.28)$ : $\mathrm{C}, 61.99 ; \mathrm{H}, 4.83 ; \mathrm{N}, 15.49$; found: $\mathrm{C}, 61.98 ; \mathrm{H}, 4.80 ; \mathrm{N}, 15.47 \%$. ${ }^{1} \mathrm{H}$ NMR $\left(300 \mathrm{MHz}, \mathrm{DMSO}-d_{6}\right): \delta 10.51(\mathrm{~s}, 1 \mathrm{H}, \mathrm{NH}), 8.40(\mathrm{~s}, 1 \mathrm{H}, \mathrm{CH}), 6.89-8.08(8 \mathrm{H}, \mathrm{Ar}), 3.70\left(\mathrm{~s}, 3 \mathrm{H}, \mathrm{OCH}_{3}\right) .{ }^{13} \mathrm{C}$ NMR $\left(75 \mathrm{MHz}\right.$, DMSO-d $\left.d_{6}\right): \delta 153.5,148.8,139.2,138.6,132.9,131.7,130.5,122.0,119.7,115.1,113.8,55.7$.

(E)-1-(3-Nitrobenzylidene)-2-(p-tolyl)hydrazine (1b). Yellow solid (91\%); mp 146-150 ${ }^{\circ} \mathrm{C}$, lit $150.5{ }^{\circ} \mathrm{C} .{ }^{36} \mathrm{Anal}$. Calcd for $\mathrm{C}_{14} \mathrm{H}_{13} \mathrm{~N}_{3} \mathrm{O}_{2}(M=255.28): \mathrm{C}, 65.87 ; \mathrm{H}, 5.13 ; \mathrm{N}, 16.46$; found: $\mathrm{C}, 65.80 ; \mathrm{H}, 5.10 ; \mathrm{N}, 16.44 \% .{ }^{1} \mathrm{H} \mathrm{NMR}$ (300 MHz, DMSO- $\left.d_{6}\right): \delta 10.59(\mathrm{~s}, 1 \mathrm{H}, \mathrm{NH}), 8.41(\mathrm{~s}, 1 \mathrm{H}, \mathrm{CH}), 7.00-8.09(8 \mathrm{H}, \mathrm{Ar}), 2.22\left(\mathrm{~s}, 3 \mathrm{H}, \mathrm{CH}_{3}\right) .{ }^{13} \mathrm{C} \mathrm{NMR}(75$ $\left.\mathrm{MHz}, \mathrm{DMSO}-d_{6}\right): \delta 148.8,142.9,138.5,1335,131.8,130.5,130.1,128.5,122.2,119.8,112.7,20.7$.

(E)-1-(3-Nitrobenzylidene)-2-phenylhydrazine (1c). Yellow solid (85 \%); mp 119-120, lit 120-124 ${ }^{\circ} \mathrm{C} .{ }^{37}$ Anal. Calcd for $\mathrm{C}_{13} \mathrm{H}_{11} \mathrm{~N}_{3} \mathrm{O}_{2}(M=241.25): \mathrm{C}, 64.72 ; \mathrm{H}, 6.81 ; \mathrm{N}, 17.03$; found: $\mathrm{C}, 64.70 ; \mathrm{H}, 6.79 ; \mathrm{N}, 17.01 \% .{ }^{1} \mathrm{H} \mathrm{NMR}$ $\left(300 \mathrm{MHz}, \mathrm{DMSO}-d_{6}\right): \delta 10.70(\mathrm{~s}, 1 \mathrm{H}, \mathrm{NH}), 8.43(\mathrm{~s}, 1 \mathrm{H}, \mathrm{CH}), 6.80-8.09(9 \mathrm{H}, \mathrm{Ar}) .{ }^{13} \mathrm{C} \mathrm{NMR}\left(75 \mathrm{MHz}, \mathrm{DMSO}-d_{6}\right): \delta$ $148.8,145.2,138.3,134.2,132.0,130.6,129.7,122.4,120.0,119.9,112.7$.

(E)-1-(4-Bromophenyl)-2-(3-nitrobenzylidene)hydrazine (1d). Orange solid (94\%); mp 151-152 ${ }^{\circ} \mathrm{C}$. Anal. Calcd for $\mathrm{C}_{13} \mathrm{H}_{10} \mathrm{BrN}_{3} \mathrm{O}_{2}(M=320.15)$ : C, 48.77; $\mathrm{H}, 3.15 ; \mathrm{N}, 13.13$; found: $\mathrm{C}, 48.75 ; \mathrm{H}, 3.13 ; \mathrm{N}, 13.10 \%$. ${ }^{1} \mathrm{H} \mathrm{NMR}$ (300 $\left.\mathrm{MHz}, \mathrm{DMSO}-d_{6}\right): \delta 10.81(\mathrm{~s}, 1 \mathrm{H}, \mathrm{NH}), 8.44(\mathrm{~s}, 1 \mathrm{H}, \mathrm{CH}), 7.09-8.13(8 \mathrm{H}, \mathrm{Ar}) .{ }^{13} \mathrm{C} \mathrm{NMR}\left(75 \mathrm{MHz}, \mathrm{DMSO}-d_{6}\right): \delta 148.7$, $144.5,138.0,135.2,132.3,132.1,130.6,122.7,120.2,114.7,110.8$.

(E)-1-(4-Chlorophenyl)-2-(3-nitrobenzylidene)hydrazine (1e). Yellow (80 \%); mp 147-149 ${ }^{\circ} \mathrm{C}$, lit $153{ }^{\circ} \mathrm{C} .{ }^{36} \mathrm{Anal}$. Calcd for $\mathrm{C}_{13} \mathrm{H}_{10} \mathrm{ClN}_{3} \mathrm{O}_{2}(M=275.69)$ : $\mathrm{C}, 56.64 ; \mathrm{H}, 3.66 ; \mathrm{N}, 15.24 ;$ found: $\mathrm{C}, 56.62 ; \mathrm{H}, 3.56 ; \mathrm{N}, 15.19 \% .{ }^{1} \mathrm{H}$ NMR $\left(300 \mathrm{MHz}, \mathrm{DMSO}-d_{6}\right): \delta 10.81(\mathrm{~s}, 1 \mathrm{H}, \mathrm{NH}), 8.45(\mathrm{~s}, 1 \mathrm{H}, \mathrm{CH}), 7.14-8.13(8 \mathrm{H}, \mathrm{Ar}) .{ }^{13} \mathrm{C} \mathrm{NMR}\left(75 \mathrm{MHz}, \mathrm{DMSO}-d_{6}\right): \delta$ $148.8,144.2,138.0,135.1,132.1,130.6,129.5,123.1,122.7,120.2,114.2$.

(E)-1-(4-Fluorophenyl)-2-(3-nitrobenzylidene)hydrazine (1f). Orange solid (95\%); mp 133-135 ${ }^{\circ} \mathrm{C}$. Anal. Calcd for $\mathrm{C}_{13} \mathrm{H}_{10} \mathrm{FN}_{3} \mathrm{O}_{2}(M=259.24): \mathrm{C}, 60.23 ; \mathrm{H}, 3.89 ; \mathrm{N}, 16.21$; found: $\mathrm{C}, 60.17 ; \mathrm{H}, 3.87 ; \mathrm{N}, 16.14 \% .{ }^{1} \mathrm{H} \mathrm{NMR}(300$ $\left.\mathrm{MHz}, \mathrm{DMSO}-d_{6}\right): \delta 10.68(\mathrm{~s}, 1 \mathrm{H}, \mathrm{NH}), 8.43(\mathrm{~s}, 1 \mathrm{H}, \mathrm{CH}), 7.09-8.09(8 \mathrm{H}, \mathrm{Ar}) .{ }^{13} \mathrm{C} \mathrm{NMR}\left(75 \mathrm{MHz}, \mathrm{DMSO}-d_{6}\right): \delta 148.8$, $141.9,138.3,134.2,132.0,130.6,122.4,120.0,116.3,116.0,113.8$.

\section{Synthesis of $2 a-2 f$}

A $20 \mathrm{~mL}$ screw neck vial was charged with DMSO $(10 \mathrm{~mL})$, hydrazone $(1 \mathrm{mmol})$, tetramethylethylenediamine (TMEDA) (295 mg, $2.5 \mathrm{mmol}$ ), $\mathrm{CuCl}\left(2 \mathrm{mg}, 0.02 \mathrm{mmol}\right.$ ) and $\mathrm{CCl}_{4}$ (20 mmol, 10 equiv). After 1-3 hours (until TLC analysis showed complete consumption of corresponding Schiff base) the reaction mixture was poured into $\sim 0.01 \mathrm{M}$ solution of $\mathrm{HCl}(100 \mathrm{~mL}, \sim \mathrm{pH}=2-3)$, and extracted with $\mathrm{CH}_{2} \mathrm{Cl}_{2}(3 \times 20 \mathrm{~mL})$. The combined organic phase was washed with $\mathrm{H}_{2} \mathrm{O}(3 \times 50 \mathrm{~mL})$, brine $(30 \mathrm{~mL})$, dried over anhydrous $\mathrm{Na}_{2} \mathrm{SO}_{4}$ and concentrated in vacuo of the rotary evaporator. The residue was purified by column chromatography on silica gel using appropriate mixtures of hexane and $\mathrm{CH}_{2} \mathrm{Cl}_{2}(3 / 1-1 / 1)$, and the corresponding diazenes $\mathbf{2 a - 2} \mathbf{f}$ were obtained.

1-(2,2-Dichloro-1-(3-nitrophenyl)vinyl)-2-(4-methoxyphenyl)diazene (2a). Yellow solid (69 \%); mp 142-145 ${ }^{\circ} \mathrm{C}$. Anal. Calcd for $\mathrm{C}_{15} \mathrm{H}_{11} \mathrm{Cl}_{2} \mathrm{~N}_{3} \mathrm{O}_{3}(M=352.17)$ : $\mathrm{C}, 51.16 ; \mathrm{H}, 3.15 ; \mathrm{N}, 11.93$; found: $\mathrm{C}, 51.13 ; \mathrm{H}, 3.10 ; \mathrm{N}, 11.89 \% .{ }^{1} \mathrm{H}$ NMR (300 MHz, CDCl $): \delta 6.97-8.31(8 \mathrm{H}, \mathrm{Ar}), 3.89\left(\mathrm{~s}, 3 \mathrm{H}, \mathrm{OCH}_{3}\right) .{ }^{13} \mathrm{C} \mathrm{NMR}\left(75 \mathrm{MHz}, \mathrm{CDCl}_{3}\right): \delta 150.1,148.1$, $147.1,145.0,136.4,129.1,125.5,124.3,123.6,120.2,119.0,114.3,55.7$. ESI-MS: $\mathrm{m} / z: 353.11[M+\mathrm{H}]^{+}$. 
(E)-1-(2,2-Dichloro-1-(3-nitrophenyl)vinyl)-2-(p-tolyl)diazene (2b). Red solid (80\%); mp 140-142 ${ }^{\circ} \mathrm{C}$, lit. 141 ${ }^{\circ} \mathrm{C} .{ }^{38}$

1-(2,2-Dichloro-1-(3-nitrophenyl)vinyl)-2-phenyldiazene (2c). Red solid (70 \%); mp 125-128 ${ }^{\circ} \mathrm{C}$, lit. 125-126 ${ }^{\circ} \mathrm{C} .{ }^{8}$ Anal. Calcd for $\mathrm{C}_{14} \mathrm{H}_{9} \mathrm{Cl}_{2} \mathrm{~N}_{3} \mathrm{O}_{2}(\mathrm{M}=322.15)$ : $\mathrm{C}, 52.20 ; \mathrm{H}, 2.82 ; \mathrm{N}, 13.04$; found: $\mathrm{C}, 52.14 ; \mathrm{H}, 2.77 ; \mathrm{N}, 12.97 \%$. ${ }^{1} \mathrm{H}$ NMR $\left(300 \mathrm{MHz}, \mathrm{CDCl}_{3}\right): \delta 7.44-8.32(9 \mathrm{H}, \mathrm{Ar}) .{ }^{13} \mathrm{C} \mathrm{NMR}\left(75 \mathrm{MHz} \mathrm{CDCl}_{3}\right): \delta 152.5,150.2,148.1,144.1,139.8$, $136.4,134.2,132.1,129.2,125.4,123.7,123.3$. ESI-MS: $m / z: 322.08[M+H]^{+}$.

(E)-1-(4-Bromophenyl)-2-(2,2-dichloro-1-(3-nitrophenyl)vinyl)diazene (2d). Red solid (68 \%); mp $111-113{ }^{\circ} \mathrm{C}$. Anal. Calcd for $\mathrm{C}_{14} \mathrm{H}_{8} \mathrm{BrCl}_{2} \mathrm{~N}_{3} \mathrm{O}_{2}(M=401.04)$ : $\mathrm{C}, 41.93 ; \mathrm{H}, 2.01 ; \mathrm{N}, 10.48$; found: $\mathrm{C}, 41.86 ; \mathrm{H}, 2.01 ; \mathrm{N}, 10.38 \%$. ${ }^{1} \mathrm{H}$ NMR $\left(300 \mathrm{MHz}, \mathrm{CDCl}_{3}\right): \delta 7.53-8.32(8 \mathrm{H}, \mathrm{Ar}) .{ }^{13} \mathrm{C} \mathrm{NMR}\left(75 \mathrm{MHz}, \mathrm{CDCl}_{3}\right): \delta 162.3,151.2,150.3,148.1,137.5$, $136.3,133.9,132.5,129.3,126.8,125.3,124.8$. ESI-MS: $m / z: 402.01[M+H]^{+}$.

(E)-1-(4-Chlorophenyl)-2-(2,2-dichloro-1-(3-nitrophenyl)vinyl)diazene (2e). Red solid (64 \%); mp 109-110 ${ }^{\circ} \mathrm{C}$. Anal. Calcd for $\mathrm{C}_{14} \mathrm{H}_{8} \mathrm{Cl}_{3} \mathrm{~N}_{3} \mathrm{O}_{2}(M=356.59)$ : $\mathrm{C}, 47.16 ; \mathrm{H}, 2.26 ; \mathrm{N}, 11.78$; found: $\mathrm{C}, 47.10 ; \mathrm{H}, 2.20 ; \mathrm{N}, 11.74 \% .{ }^{1} \mathrm{H}$ NMR (300 MHz, CDCl 3 ): $\delta 7.21-8.10(8 \mathrm{H}, \mathrm{Ar}) .{ }^{13} \mathrm{C} \mathrm{NMR}\left(75 \mathrm{MHz}, \mathrm{CDCl}_{3}\right): \delta 150.7,150.1,147.9,137.849,136.3$, $133.7,132.2,130.3,129.3,125.0,124.4,123.7$. ESI-MS: $m / z: 357.34[M+H]^{+}$.

(E)-1-(2,2-Dichloro-1-(3-nitrophenyl)vinyl)-2-(4-fluorophenyl)diazene (2f). Red solid (52 \%); mp 100-103 ${ }^{\circ} \mathrm{C}$. Anal. Calcd for $\mathrm{C}_{14} \mathrm{H}_{8} \mathrm{Cl}_{2} \mathrm{FN}_{3} \mathrm{O}_{2}(M=340.14)$ : C, 49.44; $\mathrm{H}, 2.37 ; \mathrm{N}, 12.35 ;$ found: $\mathrm{C}, 49.38 ; \mathrm{H}, 2.30 ; \mathrm{N}, 12.27 \% .{ }^{1} \mathrm{H}$ NMR $\left(300 \mathrm{MHz}, \mathrm{CDCl}_{3}\right): \delta 7.12-8.33(8 \mathrm{H}, \mathrm{Ar}) .{ }^{13} \mathrm{C} \mathrm{NMR}\left(75 \mathrm{MHz}, \mathrm{CDCl}_{3}\right): \delta 151.7,146.3,143.8,136.3,129.2$, $125.6,125.4,125.4,123.8,121.3,116.4,116.1$. ESI-MS: $m / z: 341.09[M+H]^{+}$.

\section{Acknowledgements}

This work was supported by the Science Development Foundation under the President of the Republic of Azerbaijan - Grant No. EiF/MQM/EIm-Tehsil-1-2006-1(26)-71/06/4.

\section{References}

1. Zollinger H., Diazo Chemistry I: Aromatic and Heteroaromatic Compounds. New York: Wiley, 1994. https://doi.org/10.1002/3527601724

2. Zollinger H., Diazo Chemistry II: Aliphatic, Inorganic and Organometallic Compounds, VCH: Weinheim 1995. https://doi.org/10.1002/3527601732

3. Nishihara H., Coord. Chem. Rev. 2005, 249, 1468-1475. https://doi.org/10.1016/i.ccr.2004.11.009

4. Gowenlock B. G.; Richter-Addo G. B. Chem. Soc. Rev. 2005, 34, 797-809. https://doi.org/10.1039/B500855G

5. Chavan R. B., Environmentally friendly dyes, Handbook of Textile and Industrial Dyeing (Volume 1: Principles, Processes and Types of Dyes), Woodhead Publishing Limited, 2011 p. 515-561. https://doi.org/10.1533/9780857093974.2.515

6. Wang X., Azo Polymers: Synthesis, Functions and Applications, Springer-Verlag Berlin Heidelberg, 2017. https://doi.org/10.1007/978-3-662-53424-3

7. Sharma V. S.; Shah A. P.; Sharma A. S. New J. Chem. 2019, 43, 3556-3564. https://doi.org/10.1039/C8NJ04997A 
8. Nenajdenko V. G.; Shastin A. V.; Gorbachev V. M.; Shorunov S. V.; Muzalevskiy V. M.; Lukianova A. I.; Dorovatovskii P. V.; Khrustalev V. N. ACS Catal. 2017, 7, 205-209.

https://doi.org/10.1021/acscatal.6b03196

9. Mahmudov K. T.; Kopylovich M. N.; Guedes da Silva M. F. C.; Pombeiro A. J. L. Dalton Trans. 2017, 46, 10121-10138.

https://doi.org/10.1039/C7DT01685A

10. Mahmudov K. T.; Gurbanov A. V.; Aliyeva V. A.; Resnati G.; Pombeiro A. J. L. Coord. Chem. Rev. 2020, 418, 213381.

https://doi.org/10.1016/i.ccr.2020.213381

11. Cavallo G.; Metrangolo, P.; Milani, R.; Pilati, T.; Priimagi, A.; Resnati, G.; Terraneo, G. Chem. Rev. 2016, 116, 2478-2601.

https://doi.org/10.1021/acs.chemrev.5b00484

12. Mahmudov, K. T.; Gurbanov, A. V.; Guseinov. F. I.; Guedes da Silva, M. F. C. G. Coord. Chem. Rev. 2019, 387, 32-46.

https://doi.org/10.1016/i.ccr.2019.02.011

13. Mahmudov, K. T.; Kopylovich, M. N.; Guedes da Silva, M. F. C.; Pombeiro, A. J. L. Coord. Chem. Rev. 2017, 345, 54-72.

https://doi.org/10.1016/i.ccr.2016.09.002

14. Shixaliyev, N. Q.; Gurbanov, A. V.; Maharramov, A. M.; Mahmudov, K. T.; Kopylovich, M. N.; Martins; L. M. D. R. S.; Muzalevskiy, V. M.; Nenajdenko, V. G.; Pombeiro, A. J. L. New J. Chem. 2014, 38, 4807-4815. https://doi.org/10.1039/C4NJ00797B

15. Shixaliyev N.Q., Maharramov A.M., Gurbanov A.V., Nenajdenko V.G., Muzalevskiy V.M., Mahmudov K.T., Kopylovich M.N. Catal. Today 2013, 217, 76-79.

https://doi.org/10.1016/j.cattod.2013.06.013

16. Lim J.Y.C., Beer P.D. Chemistry 2018, 4, 731-783.

https://doi.org/10.1016/i.chempr.2018.02.022

17. Roithová J. Chem. Soc. Rev. 2012, 41, 547-559.

https://doi.org/10.1039/C1CS15133A

18. Shixaliyev, N. Q.; Ahmadova, N. E.; Gurbanov, A. V.; Maharramov, A. M.; Mammadova, G. Z.; Nenajdenko, V. G.; Zubkov, F. I.; Mahmudov, K. T.; Pombeiro, A. J. L. Dyes Pigments 2018, 150, 377-381. https://doi.org/10.1016/i.dyepig.2017.12.033

19. Maharramov, A. M.; Shikhaliyev, N. Q.; Suleymanova G. T.; Gurbanov, A. V. Babayeva, G. V.; Mammadova, G.Z.; Zubkov F.I.; Nenajdenko V.G.; Mahmudov K.T.; Pombeiro A.J.L. Dyes Pigments 2018, 159, $135-141$. https://doi.org/10.1016/j.dyepig.2018.06.022

20. Shikhaliyev N.Q.; Kuznetsov M.L.; Maharramov A.M.; Gurbanov A.V.; Ahmadova N.E.; Nenajdenko V.G.; Mahmudov K.T.; Pombeiro A.J.L. CrystEngComm 2019, 21, 5032-5038. https://doi.org/10.1039/C9CE00956F

21. Tskhovrebov A.G.; Solari E.; Scopelliti R.; Severin K. Organometallics 2014, 33, 2405-2408. https://pubs.acs.org/doi/abs/10.1021/om500333y

22. Tskhovrebov A.G.; Vasileva A.A.; Goddard R.; Riedel T.; Dyson P.J.; Mikhaylov V.N.; Serebryanskaya T.V.; Sorokoumov V.N.; Haukka M. Inorg. Chem. 2018, 57, 930-934. https://pubs.acs.org/doi/abs/10.1021/acs.inorgchem.8b00072

23. Bondi, A. J. Phys. Chem. 1964, 68, 441-451.

https://doi.org/10.1021/j100785a001 
24. Marcus, Y. J. Sol. Chem. 1991, 20, 929-944.

https://doi.org/10.1007/BF01074953

25. Hansch C.; Leo A.; Taft W. R. Chem. Rev. 1991, 91, 165-195.

https://doi.org/10.1021/cr00002a004

26. Maharramov, A. M.; Aliyeva, R. A.; Aliyev, I. A.; Pashaev, F. G.; Gasanov, A. G.; Azimova, S. I.; Askerov, R. K.; Kurbanov, A. V.; Mahmudov K. T. Dyes Pigments 2010, 85, 1-6.

https://doi.org/10.1016/i.dyepig.2009.09.003

27. Mahmudov, K. T.; Maharramov, A. M.; Aliyeva, R. A.; Aliyev, I. A.; Askerov, R. K.; Batmaz, R.; Kopylovich, M. N.; Pombeiro, A. J. L. J. Photochem. Photobiol. A: Chem. 2011, 219, 159-165.

https://doi.org/10.1016/i.jphotochem.2011.02.006

28. Reichardt, C. Chem. Rev. 1994, 94, 2319-2358.

https://doi.org/10.1021/cr00032a005

29. Mahmudov, K. T.; Maharramov, A. M.; Aliyeva, R. A.; Aliyev, I. A.; Kopylovich, M. N.; Pombeiro, A .J. L. Anal. Lett. 2010, 43, 2923-2938.

https://doi.org/10.1080/00032711003763665

30. Asadov, Z. H.; Rahimov, R. A.; Ahmadova, G. A.; Mammadova, K. A.; Gurbanov, A .V. J. Surfact. Deterg.

2016, 19, 145-153.

https://doi.org/10.1007/s11743-015-1762-y

31. Gurbanov, A. V.; Maharramov, A. M.; Zubkov, F. I.; Saifutdinov, A. M.; Guseinov, F. I. Aust. J. Chem. 2018, 71, 190-194.

https://doi.org/10.1071/CH17595

32. Afkhami, F. A.; Khandar, A. A.; Mahmoudi, G.; Maniukiewicz, W.; Gurbanov, A. V.; Zubkov, F. I.; Şahin, O.; Yesilel, O. Z.; Frontera, A. CrystEngComm. 2017, 19, 1389-1399.

https://doi.org/10.1039/C6CE02666D

33. Mahmoudi, G.; Dey, L.; Chowdhury, H.; Bauzá, A.; Ghosh, B. K.; Kirillov, A. M.; Seth, S. K.; Gurbanov, A. V. Inorg. Chim. Acta 2017, 461, 192-205.

https://doi.org/10.1016/i.ica.2017.02.021

34. Gurbanov, A. V.; Mahmoudi, G.; Guedes da Silva, M. F. C.; Zubkov, F. I.; Mahmudov, K. T.; Pombeiro, A. J. L. Inorg. Chim. Acta 2018, 471, 130-136.

https://doi.org/10.1016/i.ica.2017.10.042

35. Mahmoudi, G.; Khandar, A. A.; Afkhami, F. A.; Mirslaw, B.; Gurbanov, A. V.; Zubkovm F. I.; Kennedy, A.;

Franconetti, A.; Frontera, A., CrystEngComm 2019, 21, 108-117.

https://doi.org/10.1039/C8CE01580E

36. Chattaway, C. J. Chem. Soc. 1923, 123, 3059.

https://doi.org/10.1039/CT9232302999

37. Dadiboyena, S.; Valente, E. J.; Hamme II, A. T. Tetrahedron Lett. 2009, 50, 291-294.

https://doi.org/10.1016/i.tetlet.2008.10.145

38. Shikhaliyev, N. G.; Suleymanova, G. T.; İsrayilova, A. A.; Ganbarov, K. G.; Babayeva, G. V.; Garazadeh, K. A.; Mammadova, G. Z.; Nenajdenko, V. G. Arkivoc 2019, (vi), 64-73.

https://doi.org/10.24820/ark.5550190.p010.979

This paper is an open access article distributed under the terms of the Creative Commons Attribution (CC BY) license (http://creativecommons.org/licenses/by/4.0/ 